\title{
Mean-field and blocking effects on odd-even mass differences and rotational motion of nuclei
}

\author{
F. R. Xu, ${ }^{1, *}$ R. Wyss, ${ }^{2}$ and P. M. Walker ${ }^{1}$ \\ ${ }^{1}$ Department of Physics, University of Surrey, Guildford, Surrey GU2 5XH, England \\ ${ }^{2}$ Department of Physics, Royal Institute of Technology, Frescativägen 24, S-104 05 Stockholm, Sweden
}

(Received 24 May 1999; published 27 September 1999)

\begin{abstract}
The odd-even differences of nuclear masses are strongly influenced by mean-field and odd-nucleon blocking effects. When such effects are taken into account, the determination of the pairing interaction strength needs to be modified, resulting in larger pairing gaps. This method leads to an improved description for both moments of inertia and backbending frequencies of rotational bands, with no additional parameters.

[S0556-2813(99)50111-8]

PACS number(s): 21.60.Cs, 21.10.Dr, 27.70.+q
\end{abstract}

Since the Bardeen-Cooper-Schrieffer (BCS) theory was applied to atomic nuclei $[1,2]$, pairing correlations have been crucial to the understanding of many properties, such as binding energies, collective rotational motion, and quasiparticle excitation energies. The interaction strength, $G$, of the pairing force is the key parameter that governs the properties of the short-range correlations.

The $G$ value is usually determined by fitting the BCS pairing gaps ( $\Delta=G \Sigma_{i} U_{i} V_{i}$ [2]) of even-even nuclei to experimental odd-even mass differences. However, if one then calculates the corresponding theoretical mass differences (i.e., in the same manner as calculating the experimental value, but with theoretical masses), it turns out that they are systematically smaller than the experimental mass differences and also smaller than the BCS pairing gaps, at least for the deformed rare-earth nuclei described below. The experimental mass difference should, in principle, be identical to the corresponding theoretical value, but not to the pairing gap, though the gap plays the dominant role in determining the mass difference.

The above systematic discrepancies suggest that other significant effects exist. It has been pointed out, in a recent work by Satuła, Dobaczewski, and Nazarewicz [3], that one of the important effects stems from the deformed mean field. Due to the twofold Kramers degeneracy of single-particle levels, odd- and even-nucleon systems in the deformed field have different energies, which contribute to odd-even mass differences. For light- and medium-mass nuclei, the Kramers effect can be comparable with the pairing contribution [3]. Furthermore, when neighboring nuclei have different deformations, the shape-changing effect also plays a role. These two factors originate from the deformed mean field and, therefore, we will refer to them in the following as the meanfield effect.

Moreover, the pairing gaps of even-even nuclei cannot include the odd-nucleon blocking effects of adjacent odd nuclei, while experimental odd-even mass differences of course contain such blocking effects $[2,4]$. This can become significant when the density of single-particle levels around the

\footnotetext{
*Permanent address: Department of Technical Physics, Peking University, Beijing 100871, China.
}

Fermi surface is not very high. Simple BCS calculations typically show that the odd-nucleon blockings can reduce pairing gaps by more than $10 \%$ for the rare-earth nuclei. Hence, it should be expected that the mean-field and blocking effects influence the determination of pairing strengths.

Two very sensitive probes of pairing correlations and, therefore, of the pairing strengths, are moments of inertia (see, e.g., $[2,5,6]$ ) and backbending (bandcrossing) frequencies $[7,8]$. High-seniority states may serve as another probe. The recent calculations of the energies of multiquasiparticle states show the need for the adjustment of pairing strengths [9]. Hence, the question arises as to whether the pairing strength determined from odd-even mass differences is consistent with the pairing strength used to calculate moments of inertia or the energies of high-seniority states. This is an important issue for the quantitative description of nuclear properties. In this paper, we show that when the mean-field and blocking effects are taken into account, the pairing strengths need to be modified in order to reproduce the oddeven mass differences. Such modifications result in an improved description for both moments of inertia and bandcrossing frequencies.

According to the Strutinsky energy theorem [10], the total energy of a nucleus can be decomposed into a macroscopic and a microscopic part. The latter consists of shell and pairing correction energies. For the macroscopic energy, we employ the standard liquid-drop model of Ref. [11]. The microscopic energy is calculated within the deformed WoodsSaxon (WS) model $[12,13]$. The pairing correlations are treated by a technique of approximate particle-number projection, known as the Lipkin-Nogami (LN) method [14], which takes particle-number-fluctuation effects into account by introducing an additional Lagrange multiplier, $\lambda_{2}$. Both monopole and quadrupole pairings are included.

The odd-even mass difference, $D^{\text {oe }}$, can be expressed by a three-point (see, e.g., [3]), a four-point [2], or a five-point [15] formula with respect to nuclear masses. Considering that nuclear masses contain nonlinear terms in nucleon number, such as the symmetry and Coulomb energies [11], which cannot be well canceled out in the three-point formula [3], we use the five-point formula to minimize the influences of the quantities that are not relevant for the present discussion. For an even-even nucleus [15], 


$$
\begin{aligned}
D^{\mathrm{oe}}= & -\frac{1}{8}[M(N+2)-4 M(N+1)+6 M(N)-4 M(N-1) \\
& +M(N-2)],
\end{aligned}
$$

where $M(N)$ is the mass of an atom with neutron number, $N$ (or $Z$ for protons). The quantity, $D^{\mathrm{oe}}$, is calculated along an isotopic (or isotonic) chain. Using the above formula, the smooth nonlinear terms can be canceled, up to the fourth order $[15,16]$ when neglecting the shape-changing effect.

The nuclear shape is determined by minimizing the calculated potential energy surface (PES) in the quadrupole deformation $\left(\beta_{2}, \gamma\right)$ space with hexadecapole $\left(\beta_{4}\right)$ variation. For well-deformed nuclei, pairing energies only weakly influence the values of deformations $[9,10]$. In the determination of equilibrium deformations, we use the monopole pairing strength obtained by the average gap method [16]. The quadrupole pairing strength is determined by restoring the local Galilean invariance with respect to quadrupole shape oscillations $[17,18]$. Whereas quadrupole pairing is important for the proper description of the moments of inertia $[19,20]$, its influence on nuclear binding energies is negligible, since we use the doubly stretched quadrupole operators $[17,18]$.

For the total energy of a nucleus, the shell-correction term is sensitive to the level distribution around the Fermi surface, which is strongly related to the particle number and deformation of the nucleus [10]. It is via the shell energies that the Kramers degeneracy affects odd-even mass differences [3]. On the other hand, macroscopic surface and Coulomb energies are deformation dependent [11], which can also contribute to the $D^{\text {oe }}$ values if considering shape changes with particle number. These effects originate from the deformed mean field. The mean-field effect can be calculated using Eq. (1) with only the shell and macroscopic energies included for the quantity $M$ of the equation. The results show that the mean-field effects are usually of the order of 100 to $200 \mathrm{keV}$ for even-even rare-earth nuclei. If one neglects the changes of nuclear deformation, the Kramers effect for a deformed even system $(N=2 n)$ can be written as $\frac{1}{2}\left(e_{n+1}-e_{n}\right)$ for the three-point formula [3] or $\frac{1}{4}\left(e_{n+1}-e_{n}\right)$ for the five-point formula (where $e_{i}$ is the single-particle energy). However, the Kramers effect obtained from the above simple forms differs from the corresponding value calculated according to Eq. (1) when shape changes are included. This implies that the polarization effects of the odd nucleons have to be considered explicitly, as is done in the present work.

In the LN model (for the case of monopole pairing) the quantity, $\Delta+\lambda_{2}$, is assumed to be identical [16] with oddeven mass difference, provided that other physical influences (e.g. the mean-field and blocking effects) are ignored. In order to extract the blocking effect, we define an odd-even pairing-energy difference, $D_{p}^{\mathrm{oe}}$. The $D_{p}^{\mathrm{oe}}$ value is calculated using Eq. (1) but with only the pairing energies included for the quantity $M$ in Eq. (1). In the pairing calculations of odd nuclei, the odd-nucleon blockings are taken into account [9]. The blocking effect can be extracted by

$$
\delta_{\text {block }}=D_{p}^{\mathrm{oe}}-\left(\Delta+\lambda_{2}\right)
$$
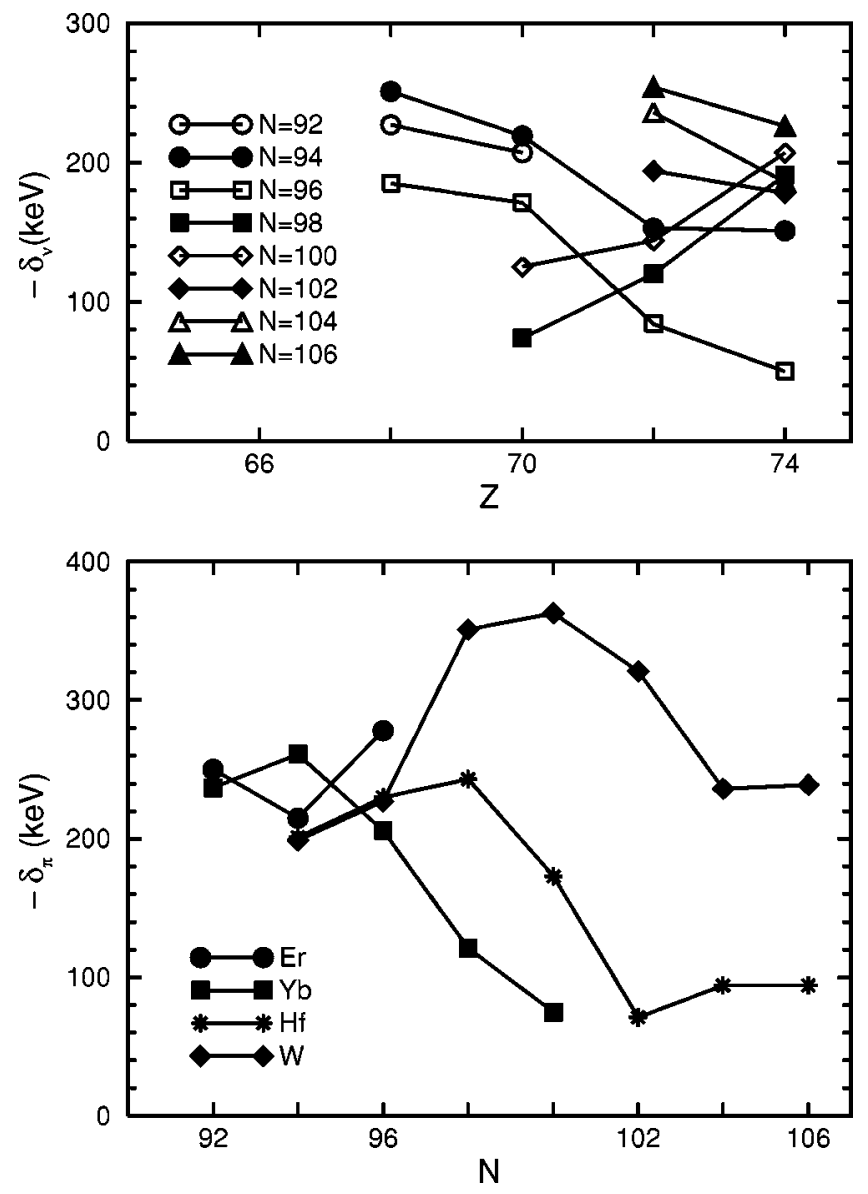

FIG. 1. Obtained mean-field and blocking effects $\left(\delta=\delta_{\mathrm{MF}}\right.$ $\left.+\delta_{\text {block }}\right)$ as a function of nucleon number. The upper panel is for neutrons $(\nu)$ and the lower panel is for protons $(\pi)$. Note that the $\delta$ values are negative.

If the blocking effect were neglected, we should have $D_{p}^{\text {oe }}$ $\approx \Delta+\lambda_{2}$. Since both the $D_{p}^{\text {oe }}$ and $\Delta+\lambda_{2}$ values increase (decrease) with increasing (decreasing) pairing strength, the $\delta_{\text {block }}$ value is not very sensitive to the change in the $G$ value. We calculate the blocking effect with the $G$ value obtained by the average gap method [16]. The results show that the values of the blocking effect, $\delta_{\text {block }}$, are usually about -200 to $-400 \mathrm{keV}$ for deformed rare-earth nuclei. It can be seen that there is partial cancellation between the mean-field and the blocking effects, but nonzero effects remain systematically.

We are interested in the well-deformed rare-earth nuclei where an abundance of regular collective rotational bands with backbendings have been observed. The combined effect ( $\delta=\delta_{\mathrm{MF}}+\delta_{\text {block }}$ ) from the mean field $\left(\delta_{\mathrm{MF}}\right)$ and blocking ( $\left.\delta_{\text {block }}\right)$ is shown in Fig. 1 . The $\delta$ values range mostly from -100 to $-300 \mathrm{keV}$, or about 10 to $30 \%$ of the corresponding odd-even mass differences, clearly suggesting that one cannot neglect this component.

In general, the mean-field and blocking effects change smoothly with particle number, and one would like to separate their contributions. However, the situation can become rather complex in some cases. When $N=98-102$, for example, the calculated PES's show that the nuclei are soft in 
the $\beta_{2}$ deformation, particularly for ${ }^{172-176} \mathrm{~W}$. The $\beta_{2}$ softness results in relatively large uncertainties in the determination of the $\beta_{2}$ values and hence significantly influences the $\delta_{\mathrm{MF}}$ values. The same holds for the $\delta_{\text {block }}$ values, leading to fluctuating results. Hence, separate consideration of the $\delta_{\mathrm{MF}}$ and $\delta_{\text {block }}$ values can be misleading. In contrast, the combined value of the mean-field and the blocking effects is less shape dependent, since the total energy of a soft nucleus is not so sensitive to the deformation value in an area around the minimum of its PES.

With inclusion of the above mean-field and blocking effects, $\delta$, the theoretical odd-even mass difference, $D_{\text {th }}^{\text {oe }}$, can be written as

$$
D_{\mathrm{th}}^{\mathrm{oe}}=\Delta+\lambda_{2}+\delta
$$

In the practical calculations, the contribution from quadrupole pairing is also included, though this term is not written explicitly in the above equation. However, as mentioned, the contribution of the doubly stretched quadrupole pairing energies is very small (usually less than $30 \mathrm{keV}$ in magnitude). On the right-hand side of Eq. (3) the pairing gap, $\Delta$, is the dominant term. Obviously, due to the presence of the $\delta$ term, the $D_{\text {th }}^{\text {oe }}$ is, in general, not equivalent to the quantity, $\Delta$ $+\lambda_{2}$, of the even-even nucleus. The $\Delta$ value is very sensitive to the $G$ value, while the $\lambda_{2}$ and $\delta$ values are not.

The presence of the negative $\delta$ values implies that the pairing strength, $G$, needs to be increased when one aims at self-consistent calculations of odd-even mass differences. By adjusting the pairing strength, one can reproduce the experimental $D^{\text {oe }}$ value with Eq. (3). However, in that case we found that each nucleus requires a separate determination of its $G$ value. Apparently, the average gap method [16] does not give proper particle-number and deformation dependence for the pairing gaps of the studied nuclei. On the other hand, other quantities contributing to the odd-even mass difference may be lacking in our model. One such effect is the coupling to phonons, which will influence the ground-state binding energy, depending on the softness of the nuclear shape. Also, displacements of the single-particle spectrum of the WoodsSaxon potential will affect the calculated $D^{\text {oe }}$ values. To disentangle the different contributions, especially to optimize a method to determine the average pairing strength, is outside the scope of the present work.

We scale the pairing strength by $G=F G^{0}$, where $G^{0}$ is the strength obtained by the average gap method [16]. For reasons of simplicity, we use a constant $F$ factor for the local mass region of the studied nuclei. The constant $F$ value is determined by averaging the individual $F$ values that have been obtained by reproducing the corresponding $D_{\text {expt }}^{\text {oe }}$ values of the nuclei. We expect that using the average $F$ value will reduce the fluctuations arising from the uncertainties of experimental masses and from possible discrepancies between theoretical and experimental single-particle levels. For the region of the studied nuclei, we obtain $\widetilde{F}_{\nu}=1.08$ (neutrons) and $\widetilde{F}_{\pi}=1.05$ (protons) using the experimental masses of [21]. This results in increases of the LN pairing gaps by about $25 \%$ for neutrons and $15 \%$ for protons.
To investigate the consistency of our method, we calculate the moments of inertia of yrast rotational bands by means of the pairing-deformation self-consistent cranked shell model $[22,23]$. In this model, pairing and deformation change with rotational frequency in a self-consistent way, i.e., for a given frequency, pairing is self-consistently treated by solving the cranked LN equation, and deformation is determined by minimizing the calculated total Routhian surface (TRS) [22]. The total collective angular momentum is calculated as follows:

$$
I_{x}=\sum_{\alpha, \beta>0}\left\langle\beta\left|\hat{j}_{x}\right| \alpha\right\rangle \rho_{\alpha \beta}+\sum_{\alpha, \beta>0}\left\langle\widetilde{\beta}\left|\hat{j}_{x}\right| \widetilde{\alpha}\right\rangle \rho_{\alpha \tilde{\beta}}
$$

where $\rho$ is the density matrix of the cranked LN model in the representation of signature basis denoted explicitly by $\alpha$, $\beta$ ( $\widetilde{\alpha}, \widetilde{\beta}$ are for opposite signatures) [22]. The moment of inertia is then obtained by $J^{(1)}=I_{x} / \omega$; here $\omega$ is the rotational frequency.

As mentioned earlier, the moment of inertia is a very sensitive probe of pairing correlations. It is not at all obvious that a pairing interaction which reproduces the odd-even mass difference can, at the same time, also reproduce the moment of inertia. In Fig. 2, we compare the experimentally deduced moments of inertia with our results calculated with the "standard" strength $G^{0}$ and the adjusted $G=\widetilde{F} G^{0}$. Clearly, the adjusted $G$ values lead to an improved description for both moments of inertia and backbending frequencies. (No additional parameters are involved or adjusted.)

In this context, one needs to recall the long-standing problem of cranking calculations in the case of monopole pairing, i.e., one cannot at the same time describe both moments of inertia and bandcrossing frequencies (see, e.g., [7,8]). In order to reproduce moments of inertia, one in general needs to use a reduced pairing strength $[6,8]$. On the other hand, an enhanced strength is required to reproduce bandcrossing frequencies [7]. The presence of the time-odd component of the quadrupole pairing field induces an additional contribution to the moment of inertia [19,20], which allows an increase in the $G$ value. Apparently, the doubly stretched quadrupole pairing interaction, in combination with the cranked LN method, enables a consistent discription for both moments of inertia and bandcrossing frequencies. However, other effects, such as the coupling with vibrations, may also affect the crossing frequencies.

For some heavy isotopes, using the average $\widetilde{F}$ value results in too-small moments of inertia, e.g., in ${ }^{178} \mathrm{Hf}$ and ${ }^{180} \mathrm{~W}$. For these isotopes, the average $\widetilde{F}$ value gives too-large $D_{\text {th }}^{\text {oe }}$ values compared to the corresponding $D_{\text {expt }}^{\text {oe }}$ values. In fact, the $\Delta+\lambda_{2}$ values obtained from the "standard" $G^{0}$

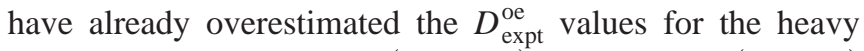
isotopes, e.g., by $166 \mathrm{keV}$ (neutrons) and $190 \mathrm{keV}$ (protons) in ${ }^{178} \mathrm{Hf}$ and, correspondingly, $98 \mathrm{keV}$ and $125 \mathrm{keV}$ in ${ }^{180} \mathrm{~W}$. In general, the average gap method gives too-large $\Delta+\lambda_{2}$ values for heavy isotopes and too-small $\Delta+\lambda_{2}$ values for light isotopes, indicating the problem of the $A$ dependence of the pairing gaps. Obviously, averaging the $G$-adjusting factors does not change the $A$ dependence. 


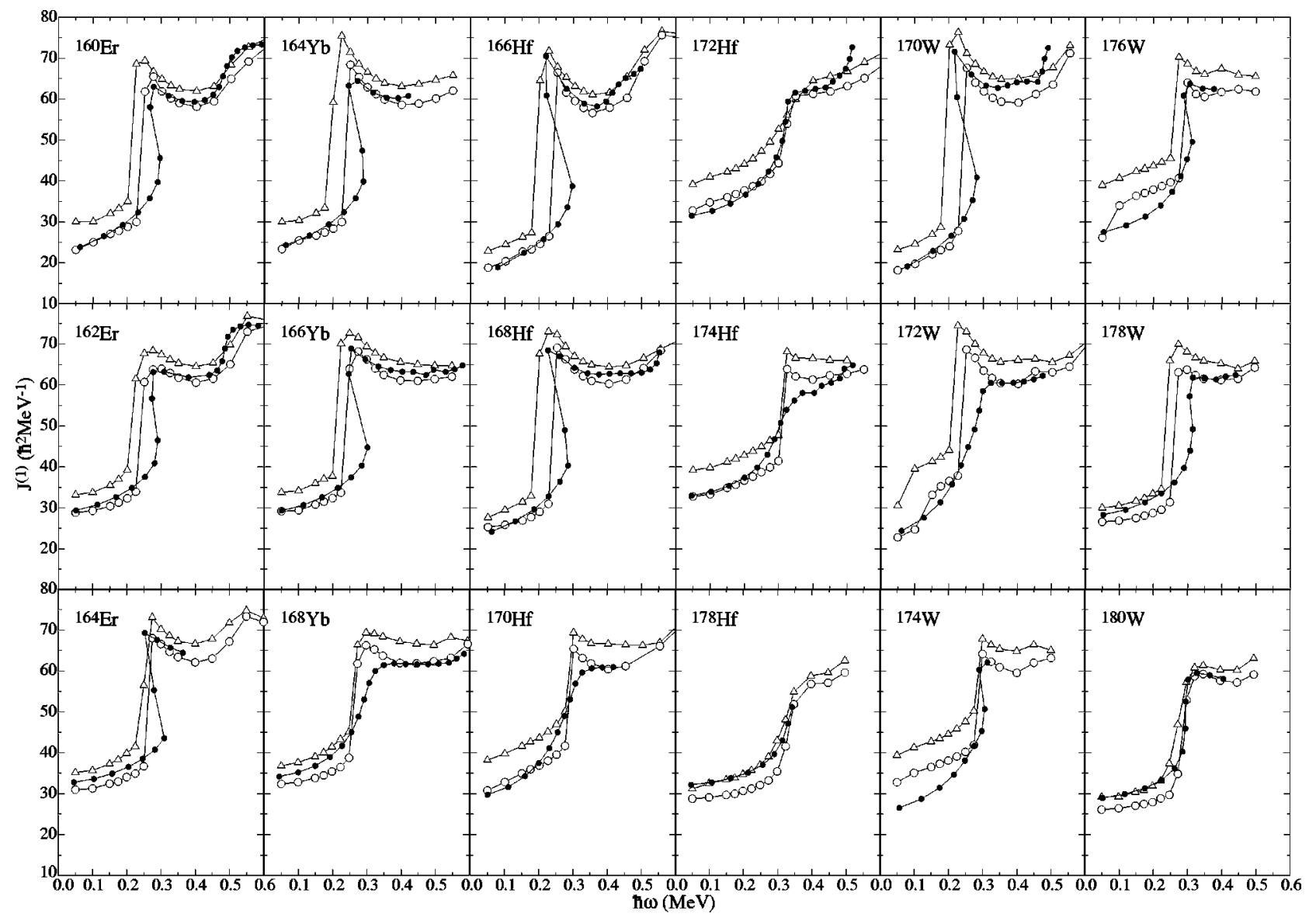

FIG. 2. Calculated and experimental moments of inertia. The open triangles and circles denote the calculations with the pairing strengths obtained by the average gap method $\left(G^{0}\right)$ and those adjusted, on average, for mean-field and blocking effects $\left(G=\widetilde{F} G^{0}\right)$, respectively. The dots show the experimental values [24].

In order to check the influence of the above unsatisfactory $A$ dependence, we have also done the calculation with the pairing strength, $G_{0}$, which makes $\Delta+\lambda_{2}=D_{\text {expt }}^{\text {oe }}$ for each given nucleus. The $G_{0}$ value is normally smaller than the "standard" $G^{0}$ value for a heavy isotope, e.g., in ${ }^{178} \mathrm{Hf}$ and ${ }^{180} \mathrm{~W}$. Results show that the moments of inertia obtained with such $G_{0}$ values are systematically larger than the corresponding experimental moments of inertia. However, when the $G_{0}$ value instead is adjusted by reproducing the $D_{\text {expt }}^{\text {oe }}$ value with $\Delta+\lambda_{2}+\delta$ (i.e., including the mean-field and blocking effects, $D_{\text {th }}^{\text {oe }}=D_{\text {expt }}^{\text {oe }}$ ) a significantly improved description can be obtained, as shown in Fig. 3 for ${ }^{178} \mathrm{Hf}$ and ${ }^{180} \mathrm{~W}$. Here we have obtained different $F$ values (see Fig. 3, caption) compared to the above average $\widetilde{F}$ value, mainly because the different pairing strengths $\left(G^{0}\right.$ or $\left.G_{0}\right)$ have been chosen as the reference of the $G$ adjustment. The nonaverage $F$ values are mostly in the range of $1.05-1.10$ for neutrons and 1.03-1.08 for protons. Clearly, the proper pairing strength for odd-even mass differences is also consistent with that for moments of inertia. In addition, the increase of the pairing strength found in the present work agrees with that needed to reproduce the excitation energies of high-seniority states [9].

The moment of inertia is also sensitive to nuclear shape. In order to check the determined deformations, we calculated intrinsic quadrupole moments [9], and obtained results which agree with the corresponding experimental values [25]. The deformation changes due to the adjustments of the $G$ values are very small $\left(\left|\Delta \beta_{2}\right|<0.003\right.$ and $\left.\left|\Delta \beta_{4}\right| \leqslant 0.002\right)$ for nuclei

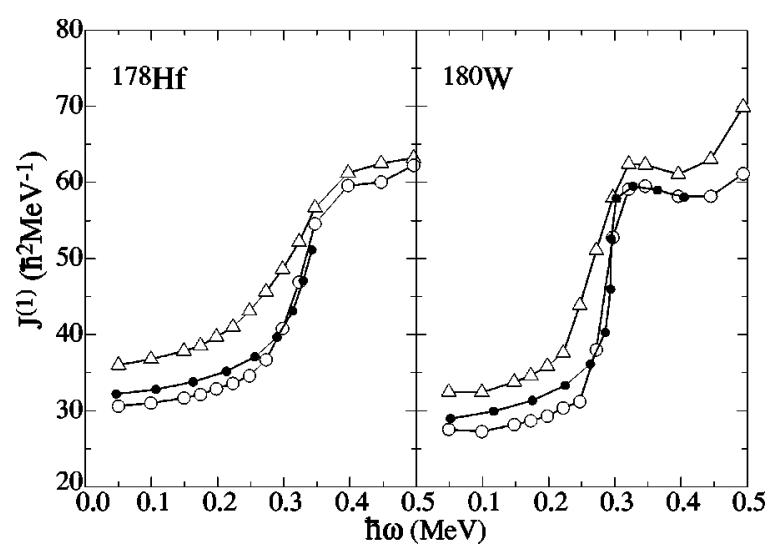

FIG. 3. Similar to Fig. 2, but with nonaverage $G$ values. The open triangles are for the calculations with the $G_{0}$ values, which make $\Delta+\lambda_{2}=D_{\text {expt }}^{\mathrm{oe}}$ for each nucleus. The open circles show the results with $G / G_{0}=1.13$ (neutons) and 1.05 (protons) for ${ }^{178} \mathrm{Hf}$ and, correspondingly, 1.10 and 1.09 for ${ }^{180} \mathrm{~W}$, which make $\Delta+\lambda_{2}$ $+\delta=D_{\text {expt }}^{\text {oe }}$ (i.e., including the mean-field and blocking effects). 
that are not soft. In ${ }^{172,176} \mathrm{~W}$, some shifts in $J^{(1)}$ are seen, which are due to the shifts of the $\beta_{2}$ values with increasing rotational frequency. For ${ }^{172-176} \mathrm{~W}$, as mentioned, the calculated TRS's are soft in the $\beta_{2}$ direction.

In summary, we have investigated the mean-field and blocking effects on odd-even mass differences for the deformed even-even nuclei in the rare-earth region. These effects are shown to be in the range of $10-30 \%$ of the corresponding odd-even mass differences, and should not be neglected in the determination of pairing strengths. Indeed, when the blocking and mean-field effects are taken into account, pairing strengths are increased by about 5-10\%, re- sulting in sizable changes of the pair gaps. The adjusted strengths are consistent with what is needed to reproduce the excitation energies of multiquasiparticle configurations, and lead to an improved description of nuclear collective rotational motion, through calculating moments of inertia and backbending frequencies. The present work establishes a consistent relation between mass differences, moments of inertia and excitation energies of high-seniority states.

This work was supported by the U.K. Engineering and Physical Sciences Research Council and the Swedish Natural Sciences Research Council.
[1] S.T. Belyaev, Mat. Fys. Medd. K. Dan. Vidensk. Selsk. 31, 1 (1959).

[2] S.G. Nilsson and O. Prior, Mat. Fys. Medd. K. Dan. Vidensk. Selsk. 32, 16 (1961).

[3] W. Satula, J. Dobaczewski, and W. Nazarewicz, Phys. Rev. Lett. 81, 3599 (1998).

[4] J.Y. Zeng and T.S. Cheng, Nucl. Phys. A405, 1 (1983).

[5] R. Bengtsson and S. Ảberg, Phys. Lett. B 172, 277 (1986).

[6] L. Goetting and W. Nazarewicz, J. Phys. G 16, L51 (1990).

[7] R. Bengtsson, Y.S. Chen, J.-Y. Zhang, and S. Åberg, Nucl. Phys. A405, 221 (1983).

[8] T. Bengtsson, Nucl. Phys. A496, 56 (1989).

[9] F.R. Xu, P.M. Walker, J.A. Sheikh, and R. Wyss, Phys. Lett. B 435, 257 (1998).

[10] M. Brack, J. Damgaard, A.S. Jensen, H.C. Pauli, V.M. Strutinsky, and C.Y. Wong, Rev. Mod. Phys. 44, 320 (1972).

[11] W.D. Myers and W.J. Swiatecki, Ann. Phys. (N.Y.) 55, 395 (1969).

[12] W. Nazarewicz, J. Dudek, R. Bengtsson, T. Bengtsson, and I.
Ragnarsson, Nucl. Phys. A435, 397 (1985).

[13] A. Oros, I. Wiedenhöver, and R. Wyss (unpublished).

[14] H.C. Pradhan, Y. Nogami, and J. Law, Nucl. Phys. A201, 357 (1973).

[15] D.G. Madland and J.R. Nix, Nucl. Phys. A476, 1 (1988).

[16] P. Möller and J.R. Nix, Nucl. Phys. A536, 20 (1992).

[17] H. Sakamoto and T. Kishimoto, Phys. Lett. B 245, 321 (1990); Nucl. Phys. A501, 205 (1989).

[18] W. Satula and R. Wyss, Phys. Rev. C 50, 2888 (1994).

[19] M. Diebel, Nucl. Phys. A419, 221 (1984).

[20] R. Wyss and W. Satula, Phys. Lett. B 351, 393 (1995).

[21] C. Audi, O. Bersillon, J. Blachot, and A.H. Wapstra, Nucl. Phys. A624, 1 (1997).

[22] W. Satula, R. Wyss, and P. Magierski, Nucl. Phys. A578, 45 (1994).

[23] W. Satula and R. Wyss, Phys. Scr. T56, 159 (1995).

[24] R.B. Firestone and V.S. Shirley, Table of Isotopes (Wiley, New York, 1996), Vol. II.

[25] S. Raman et al., At. Data Nucl. Data Tables 36, 1 (1987). 